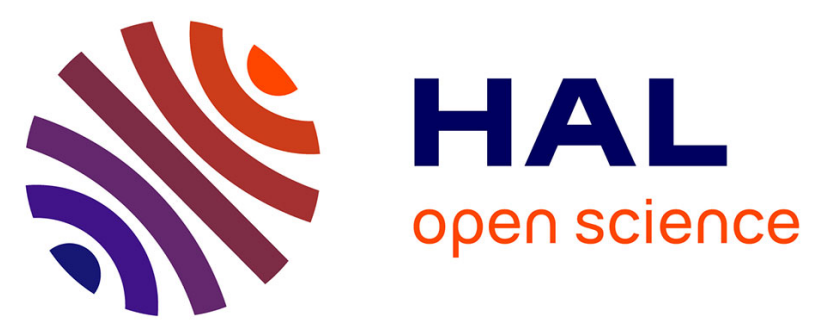

\title{
Aggregation of semifluorinated alkanes in cyclic organic solvents: A SAXS study
}

Benoît Gouze, Thibaud Nardin, Olivier Diat, Julien Cambedouzou

\section{To cite this version:}

Benoît Gouze, Thibaud Nardin, Olivier Diat, Julien Cambedouzou. Aggregation of semifluorinated alkanes in cyclic organic solvents: A SAXS study. Colloid and Interface Science Communications, 2019, 31, pp.100189. 10.1016/j.colcom.2019.100189 . hal-02270639

\section{HAL Id: hal-02270639 \\ https://hal.umontpellier.fr/hal-02270639}

Submitted on 25 Oct 2021

HAL is a multi-disciplinary open access archive for the deposit and dissemination of scientific research documents, whether they are published or not. The documents may come from teaching and research institutions in France or abroad, or from public or private research centers.
L'archive ouverte pluridisciplinaire HAL, est destinée au dépôt et à la diffusion de documents scientifiques de niveau recherche, publiés ou non, émanant des établissements d'enseignement et de recherche français ou étrangers, des laboratoires publics ou privés.

\section{(ㄷ)(1) $\$$}

Distributed under a Creative Commons Attribution - NonCommercial| 4.0 International 


\title{
Aggregation of semifluorinated alkanes in cyclic organic solvents: a SAXS study.
}

\author{
Benoît Gouze $^{1}$, Thibaud Nardin ${ }^{1,+}$, Olivier Diat ${ }^{1}$, and Julien Cambedouzou ${ }^{1,2, *}$
}

\begin{abstract}
$\underline{\text { Abstract }}$
Semifluorinated alkanes (SFA) are diblock molecules able to self-assemble in various organic media due to their fluorophilic/fluorophobic interactions. Such mixtures accordingly represent a model system for the further elaboration of soft-templated inorganic materials in totally anhydrous media. The aggregation behavior of four SFA species has been studied in two different organic and cyclic solvents in function of temperature by small angle X-ray scattering (SAXS). As long as the SFA-solvent mixtures remain in the liquid state, SAXS patterns reveal an aggregation behavior dependent on temperature and concentration, and on the internal structure of the SFA. We identify the relevant parameters governing the aggregation of these molecules based on the simulation of SAXS data versus temperature.
\end{abstract}

\section{$\underline{\text { Keywords }}$}

Small Angle X-ray Scattering, semifluorinated alkanes, self-organization, aggregation, organogels

\section{$\underline{\text { Introduction }}$}

Semifluorinated alkanes (SFA) are synthetic molecules composed of two parts: a hydrogenated alkyl chain on one hand, and a perfluorinated alkyl chain on the other hand. The usual convention in order to abbreviate the formula of SFA is FnHm, standing for a $\mathrm{F}_{3} \mathrm{C}$ $\left(\mathrm{CF}_{2}\right)_{\mathrm{n}-1}-\left(\mathrm{CH}_{2}\right)_{\mathrm{m}-1}-\mathrm{CH}_{3}$ molecule [1]. Due to the rather different physical and chemical properties of hydrogen and fluorine atoms (electronegativity, polarizability, etc.), both sides of the molecules are not compatible, therefore SFA show amphiphilic properties [2]. Consequently, SFA are expected to self-assemble in alkane (and alkane-like) liquid media in order to minimize the contact area between the fluorinated and hydrogenated parts, and therefore the system free energy [3-8]. SFA therefore constitute a fascinating model system for studying self-assembly mechanisms in non-aqueous media, and more generally in media not governed by hydrophilic/hydrophobic interactions. However, if the aggregation of SFA at liquid/air interfaces has often been described in the literature [9,10], the structure of SFA aggregates in bulk organic media is scarcely reported, and the aggregation numbers were

\footnotetext{
${ }^{+}$present address: IFP Energies nouvelles, Rond-point de l'échangeur de Solaize, BP 3, 69360 Solaize, France * corresponding author, +334 67149212
} 
usually reported as small, i.e. between 2 and 10 [2,11] and exceptionally 250 for F8H16 in hexadecane, but with huge error bars (+/- 200)[3].

As SFA molecules form gels when temperature decreases, several attempts have been made in order to describe the structure of these gel phases $[6,7,12,13]$. In particular, the aggregation behavior of F8H16 in alkanes or perfluorinated oils is certainly the most detailed one. The structure of these gels was proposed to be made of F8H16 multi-branched ribbons among mixtures of hydrogenated or fluorinated solvents [5,7]. In a recent study, we took advantage of the morphology of this gel when the liquid phase is a silicon carbide ( $\mathrm{SiC}$ ) precursor molecule in order to produce soft-templated macroporous SiC [14,15].

In an attempt to get further insight into the mechanisms governing the self-aggregation of SFA molecules, we studied the molecular structure of a number of mixtures involving different SFA, namely F8H16, F8H10, F6H14 and F6H10, and two cyclic organic molecules, namely cyclohexane $(\mathrm{CH})$ and 1,3,5-trisilacyclohexane (TSCH), with several compositions.

$\mathrm{CH}$ and TSCH are both cyclic molecules that will be referred in the following as solvents. Each molecule is made of 18 atoms including 12 hydrogen atoms and 6 atoms building the ring structure. However, in TSCH, 3 out of the 6 carbon atoms of $\mathrm{CH}$ have been substituted by silicon atoms, and therefore TSCH possesses assets for the preparation of SiC [14]. The molecular conformations of $\mathrm{CH}$ and $\mathrm{TSCH}$ are roughly the same, TSCH favor the chair one, showing slightly flattened angles with regards to $\mathrm{CH}$ because of silicon larger radii and longer Si-C bond (187 pm for Si-C against $154 \mathrm{pm}$ for C-C)[16-18]. CH and TSCH are also two apolar molecules, but the lack of electronegativity of Si compared to carbon gives TSCH some hydride-like capabilities [18].

In this article, we will describe the organization of the SFA listed above as pure, and mixed with either $\mathrm{CH}$ and $\mathrm{TSCH}$, as a function of concentration and temperature. The structure will be given in the light of small angle x-ray scattering (SAXS) data on the liquid phase and decreasing the temperature until gelation. After considering the case of the most studied F8H16 SFA, we will discriminate the effects of both the fluorinated and hydrogenated parts on the aggregation of SFA by considering mixtures of others SFA and oils. The interpretation of these data will allow us to provide key parameters for the control of the aggregation of SFA in small cyclic organic solvents, in particular in a carbosilane solvent able to be converted into $\mathrm{SiC}$. These results bring more insights in the understanding of aggregation in anhydrous media, and might also contribute to improve the control of the pore structure in mesoporous $\mathrm{SiC}$.

\section{$\underline{\text { Materials and methods }}$}

The SFA studied in this article were purchased from ABCR chemicals. Cyclohexane of purity higher than 99\% was purchased from Sigma-Aldrich. 1-3-5 trisilacyclohexane of purity higher than $95 \%$ was purchased form GELEST Inc. No further purification treatment was applied to these products. 
The liquid to gel phase transition temperatures were measured optically in a thermostatic bath. Optical microscopy images were recorded on a Zeiss microscope operating in transmission geometry, using parallel or crossed polarizers.

SAXS experiments were performed on a laboratory set-up involving a Mo anode delivering a squared beam of side $\sim 1 \mathrm{~mm}$ and of wavelength $0.710 \AA$. X-ray monochromatization was achieved using a Fox-3D® curved multishell mirror, and the collimation of the beam was performed using two sets of scatterless Ge-based slits [19]. Measurements were done in transmission geometry, and the scattering patterns were recorded using a MAR-345 twodimensional imaging plate detector. Sample conditioning involved sealed glass capillaries of diameter $2 \mathrm{~mm}$. The temperature SAXS experiments were performed using a cryo-oven involving a Peltier device and a resistive heater allowing a temperature range between $-5^{\circ} \mathrm{C}$ and $80^{\circ} \mathrm{C}$ to be accessed.

SAXS absolute intensities were deduced from experimental intensities by a standard procedure for the data treatment. The procedure involves the preliminary measurement of a calibration sample (polyethylene of known thickness $2.36 \mathrm{~mm}$ ), for which the absolute intensity is already known. The empty cell was measured, and its signal was subtracted from the sample intensity after proper normalization of both the sample and the empty cell to their respective transmission. The sample absolute intensity was then expressed in $\mathrm{cm}^{-1}$.

As the SFA sample history has an important influence on the phase transition behavior [20], all experiments were conducted according to the same procedure. Samples were first heated in order to reach the liquid state. Then, data were collected under decreasing temperature.

SAXS patterns were fitted according to the general equation giving the absolute intensity writing as follows:

$$
I(q)=N * V^{2} * \Delta \rho^{2} * P(q) * S(q)
$$

where $\mathrm{N}$ represents the number of scattering objects per volume unit, $\mathrm{V}$ stands for the volume of one object, $\mathrm{P}(\mathrm{q})$ is the form factor of the scattering object, $\mathrm{S}(\mathrm{q})$ is the structure factor and $\Delta \rho$ is the scattering contrast between the scattering objects and the continuous phase [21]. In the following, simulations of the intensity will be made using the free software SASfit [22].

The scattering length density $\rho$ for each part of SFA has been deduced from equations (2) and (3) from molar volumes data indexed in the Fedors tables [23]. They lead to molecular volumes, then to electronic density of each part, and finally to the scattering contrast, according to the following equations:

$$
\begin{aligned}
& V_{\text {molecule }}=\frac{V_{m}}{N_{a}} \\
& \rho=\frac{n_{e}}{V_{\text {molecule }}} * r_{e}
\end{aligned}
$$


with $\mathrm{V}_{\text {molecule }}$ the molecular volume, $\mathrm{V}_{\mathrm{m}}$ the molar volume determined from literature, $\mathrm{N}_{\mathrm{a}}$ the Avogadro number, $\rho$ the scattering length density of the considered part of molecule, $n_{e}$ the number of electrons and $r_{e}$ the classical electron radius $\left(2.82 * 10^{-13} \mathrm{~cm}\right)$.

In order to determine an average scattering length density of the hydrogenated phase in our sample $\rho_{\text {solv }}$, we took into account the relative volume fraction of the solvent $\varphi_{C H}$ and of the hydrogenated part of SFA $\varphi_{\mathrm{H}}$ according to equation 4 :

$$
\rho_{\text {solv }}=\rho_{C H} * \varphi_{C H}+\rho_{H} * \varphi_{H}
$$

with $\rho_{\mathrm{CH}}$ standing for the $\mathrm{CH}$ (or TSCH) scattering length density and $\rho_{\mathrm{H}}$ that of the hydrogenated part of SFA. Note that $\varphi_{C H}+\varphi_{H}=1$. The scattering length density contrast $\Delta \rho$ is then obtained from equation (5)

$$
\Delta \rho=\sqrt{\left(\rho_{F}-\rho_{\text {solv }}\right)^{2}}
$$

with $\rho_{F}$ the scattering length density of the fluorinated part of SFA. Once $\Delta \rho$ has been determined, we have considered the fluorinated parts of SFA as small and rigid cylinders dispersed into the liquid medium to build the form factor contribution. This approximation originates from both theoretical and experimental considerations based on stiffness of the chains [2]. The larger size of fluorine atoms compared to hydrogen makes the perfluorinated alkyle chains much more rigid than the hydrogenated ones. These perfluorinated chains most likely appear as cylindrical objects, an assumption that is justified through the result analysis in the discussion part. Note that when scattering vectors larger than 3-4 $\mathrm{nm}^{-1}$ are considered, the contribution from interatomic distances to the total intensity becomes non-negligible. Therefore, the agreement between experimental and calculated data is not sought for q values larger than $3 \mathrm{~nm}^{-1}$. Finally, it has to be pointed out that the fitting of such featureless scattering data implies that relative errors up to $20 \%$ should be accounted for in the size of the objects and on their relative abundance. We did not consider more than three different objects or structural sizes in our SAXS simulations in order to prevent an over-interpretation of the data. However, the fitting in absolute scale is a supplementary constraint that add some value to the procedure.

\section{$\underline{\text { Results and discussion }}$}

\section{1) Overview on pure SFA samples}

An overview of SAXS diagrams of pure SFA in the liquid state reveals three characteristic distances in the samples, as shown by the three peaks observed in all diagrams in Figure 1. Temperatures have been chosen in order to maintain a temperature gap of $\sim 25^{\circ} \mathrm{C}$ with the fusion temperature of the samples. No significant evolution of the SAXS profile with temperature was observed in the liquid state, testifying to the absence of major structural change in the medium. 


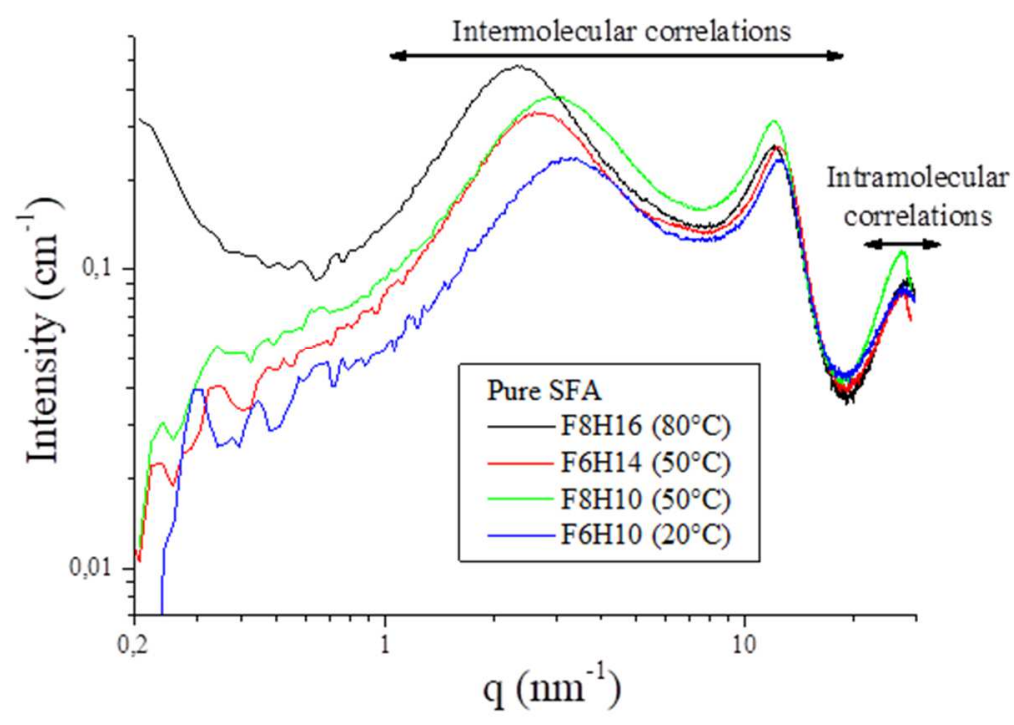

Figure 1 : SAXS diagrams of the F8H16, F6H14, F8H10, and F6H10 SFA in the liquid state

Large features appear respectively between $1-5 \mathrm{~nm}^{-1}, 10-13 \mathrm{~nm}^{-1}$ and around $28 \mathrm{~nm}^{-1}$. Each one can be related to distances between different scattering length density areas and transposed into the real space by the Bragg law:

$$
d=\frac{2 \pi}{q}
$$

where $\mathrm{d}$ is the characteristic distance in $\mathrm{nm}$ and $\mathrm{q}$ the scattering vector in $\mathrm{nm}^{-1}$. The first feature, also corresponding to the longer distance, shows different values depending on the SFA, roughly corresponding to the length of these fully extended molecules calculated using typical bond lengths, bond angles, van der Waals radii, and a specific mean contribution for the $\mathrm{CF}_{2}-\mathrm{CH}_{2}$ bound [2,24]. Rationally, the shorter distance is found for F6H10, i.e. the shortest SFA studied, and the longer distance is observed for F8H16, i.e. the longer one. The order of magnitude of the distances deduced from the SAXS patterns fits well in the range of intermolecular distances. Table SI1 sums up these measurements. The second feature, centered on $12 \mathrm{~nm}^{-1}$, roughly corresponds to the average diameters of $\mathrm{F}$ and $\mathrm{H}$ branches of the SFA when considered as fully elongated, i.e. in side-by-side configuration, in good agreement with the molecular dynamics results of Tsourtou et al. [25]. The differences between F6Hm and $\mathrm{F} 8 \mathrm{Hm}$ average diameters could be linked to the different alignment of fluorine atoms on the F branches: F6Hm being straight, while the $\mathrm{F} 8 \mathrm{Hm}$ is in a $15 / 7$ helix configuration [2]. Finally, the third feature observed above $20 \mathrm{~nm}^{-1}$ is correlated to the interatomic distances, such as between $\mathrm{CF}_{2}$ and $\mathrm{CH}_{2}$ groups in each SFA segment.

It comes out that the distances deduced from the features at the lowest scattering vectors are smaller than the fully elongated molecule length. This appears logical, since the medium is a liquid, and the $\mathrm{F}$ and $\mathrm{H}$ chains have a greater conformational freedom than in the solid state.

However, it is interesting to note that the characteristic distances observed for these four SFA can be compared to the molecular dynamics calculations by Tsourtou et al [25] on the 
F12H12 compound. In the latter model, F12H12 tend to form nanodomains of fluorinated parts or hydrogenated parts, each of them being separated from his closer identical neighbor by its counterpart. The center to center mean distances between these domains seem to be in the order of magnitude of the molecules length. Consequently, the broad feature observed around $1.5 \mathrm{~nm}^{-1}$ could be directly linked to the mean distance between these domains.

In our case, we observe that the longer the hydrogenated part of the SFA molecule, the tighter the scattering feature. The important degree of freedom of these parts allows a better organization between the domains than with smaller hydrogenated tails. This can be understood considering that longer hydrogenated tails ensure higher stability of the domains separation and reduce the disorder.

It is interesting to note that the F8H16 sample shows intensity rise at the smallest scattering vectors. The typical distances concerned are above $30 \mathrm{~nm}^{-1}$, i.e. beyond that of the intra- and intermolecular distances discussed above. It could be linked to a long-range supra-molecular organization of the domains discussed in the text, or to the presence of impurities of amorphous structure (e.g. silica particles).

To summarize, the SAXS patterns of the four pure SFA samples present similar features that can be understood in terms of intermolecular and intramolecular correlations. The first peak (centered on 1-5 $\mathrm{nm}^{-1}$ ) is related to nanodomains of fluorinated or hydrogenated parts. The second peak (10-13 $\mathrm{nm}^{-1}$ ) represents SFA side-by-side characteristic distances. Finally, the third peak around $28 \mathrm{~nm}^{-1}$ corresponds to a distance of $2.2 \AA$, in the range of intramolecular distances between $\mathrm{CF}_{2}$ and $\mathrm{CH}_{2}$ groups.

\section{2/ Aggregation of F8H16 in cyclic solvents}

We now consider mixtures of SFA with cyclic organic solvents: $\mathrm{CH}$ and TSCH. As the F8H16 SFA remains one of the most described molecules from this peculiar group, we will discuss its case in more detail than that of other SFA presented in this work. Mixing F8H16 with $\mathrm{CH}$ and $\mathrm{TSCH}$ leads to different behaviors, in particular changed gelation temperature, as shown on the phase diagrams presented in Figure 2a. Gelation can be described as the emergence of a solid fraction, forming a solid skeleton in the liquid sample. The appearance of this solid fraction was followed using optical microscopy through the appearance of birefringent inhomogeneity (Figure 2b), and SAXS through the rise of Bragg peaks in the pattern (Figure $2 \mathrm{c}$ and $2 \mathrm{~d}$ ) that are both signatures of the solid phase.

The structure of the gel phase can be described by a solid skeleton made of SFA molecules, forming ribbons or a foam-like structure in the liquid medium [26] constituted by the cyclic organic molecules. Inside the solid fraction of the gel, the arrangement is depicted as a mixture of pure lamellar and oblique lamellar lattice [12, 27], as illustrated in Figure SI1. Similar oblique lattices have been reported for F6H8 in tetradecane [8].We have studied the structure of solid pure F8H16 through SAXS measurements. The indexation of the corresponding SAXS profile is given in Figure SI2 and Table SI2. 
Focusing back on the liquid phase in F8H16/CH and F8H16/TSCH mixtures, it appears that the liquid to gel phase transition occurs at lower temperature in the case of $\mathrm{CH}$ than in $\mathrm{TSCH}$. The presence of silicon atoms in $\mathrm{TSCH}$ molecules may lower their affinity with the hydrogenated tails of F8H16 in the liquid phase, resulting in gelation at higher temperature than with $\mathrm{CH}$ molecules.

It can also be noticed from SAXS patterns that the F8H16 behavior is qualitatively the same in $\mathrm{CH}$ and $\mathrm{TSCH}$ if the temperature dependence is excluded (Figure 2a). At high enough temperature (larger than $30^{\circ} \mathrm{C}$ in $\mathrm{CH}$, larger than $0^{\circ} \mathrm{C}$ in $\mathrm{TSCH}$ ), the SAXS signal is typical of a dispersion of objects in a liquid medium. As temperature decreases, the intensity of the form factor associated to these objects increases, especially in the case where F8H16 is mixed with TSCH (black square in Figure 2d).
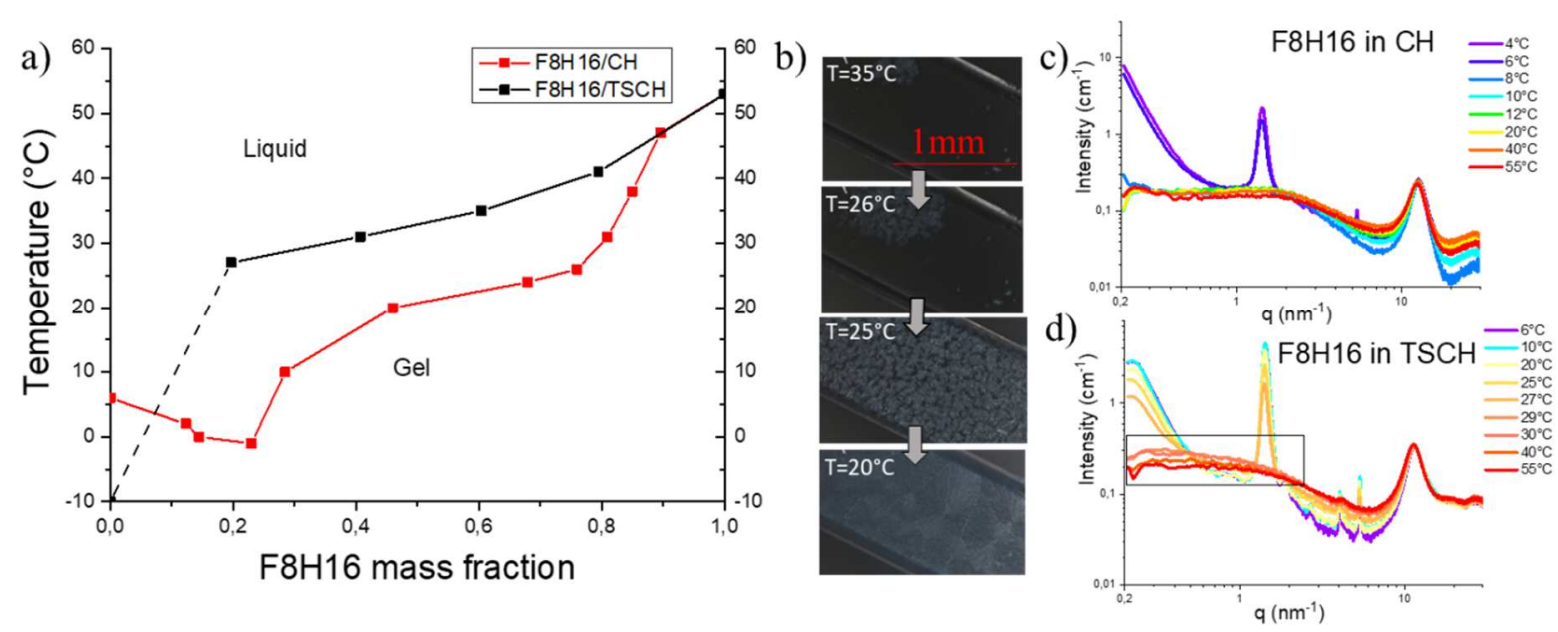

Figure 2 : F8H16 binary phase diagrams in CH and TSCH (a), optical microscopy photography between crossed polarizers of a mixture of $20 \% \mathrm{F8H} 16$ in TSCH at 35, 26, $25^{\circ} \mathrm{C}$ and $20^{\circ} \mathrm{C}$ after rest at ambient temperature (b), SAXS diagram of a $20 \% \mathrm{~F} 8 \mathrm{H} 16$ mixture in $\mathrm{CH}$ (c) and TSCH (d) as function of decreasing temperature (black rectangle enlightens the form factor evolution). The dashed line in (a) is an extrapolation of the liquid / gel transition for F8H16 weight fraction lower than 0.2 in TSCH.

Increasing intensity on the SAXS pattern can be related to an increase of the concentration, volume, and/or contrast of scattering objects in the liquid medium (equation 1), meaning that an aggregation phenomenon occurs.

As the quantity of F8H16 dispersed in $\mathrm{CH}$ or TSCH solvents increases, the SAXS patterns of the mixtures show different behaviors in function of the concentration, as seen in Figure $3 \mathrm{a}$ and $3 \mathrm{~b}$ for $\mathrm{CH}$ and $\mathrm{TSCH}$ as a solvent, respectively. A structure factor characterized by a large feature around $2 \mathrm{~nm}^{-1}$ starts to appear and gradually rises and upshits as SFA concentration increases. Characteristic distances could be extracted from these patterns and attributed to inter-object average distances, which are reduced as the F8H16 mass fraction increases.

For all F8H16 concentrations, the position of the feature associated to the structure factor always corresponds to higher distances in mixtures of SFA than in pure SFA, meaning that the solvent intercalates between the previously described domains. Note that here, the 
domains under consideration are composed by the fluorinated parts, which we intend to describe more precisely.

a)

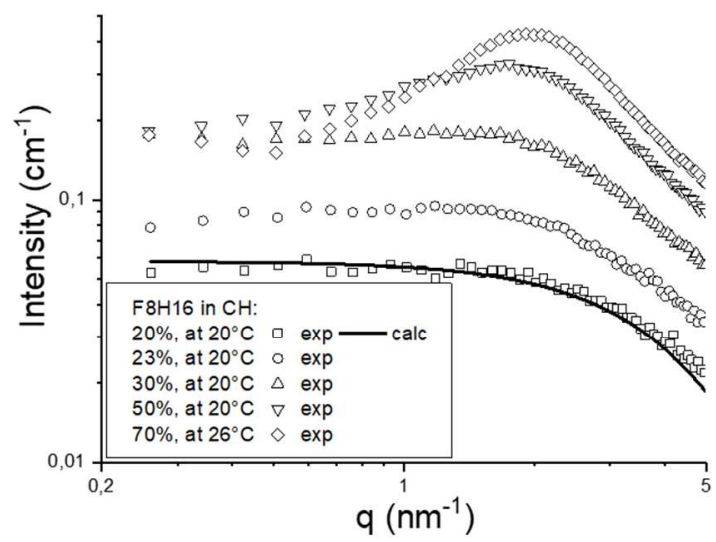

c)

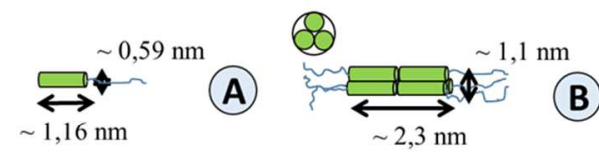

b)

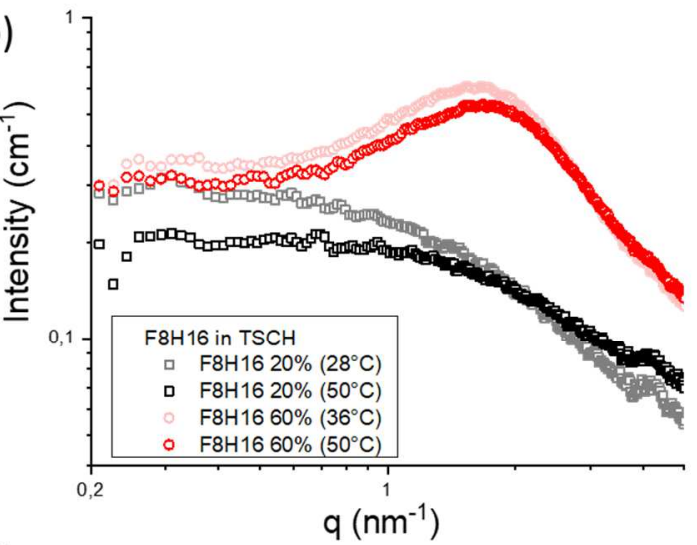

d)

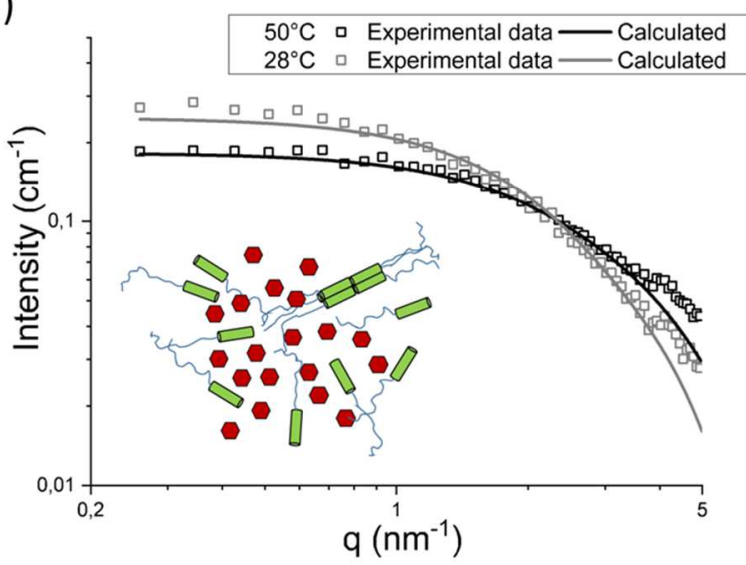

Figure 3 : SAXS patterns of mixtures of F8H16 at different concentrations in CH (a) and TSCH (b), in the liquid state. Schemes of simulated objects are represented in (c). A simulation of the SAXS diagram of a $20 \mathrm{wt} \%$ F8H16 in $\mathrm{CH}$ sample appears as the plain curve in (a). Comparison of experimental and calculated SAXS profiles of $20 \mathrm{wt} \%$

F8H16 in TSCH samples (d) with an inset representing a scheme of mixtures of fluorinated objects in green and TCSH in red.

According to the experimental SAXS data, we intend to monitor the F8H16 aggregation as a function of temperature. However, as the structure factor appearance hides the form factor of the aggregates, we have focused our work on low SFA content compositions -i.e. dilute media -, where the structure factor $S(q)$ could be taken as equal to 1 for all $q$ values.

For the $20 \mathrm{wt} . \%$ samples, the SAXS pattern shows a flat signal in the lower range of scattering vectors $\mathrm{q}$ and an intensity decrease for q values larger than $1.5 \mathrm{~nm}^{-1}$. It is typical of the form factor of dilute objects in solution, with no interactions between them.

The electronic contrast between cyclohexane (or TSCH) and the hydrogenated H16 chains, as calculated according equations 2 and 3, is very low compared to the contrast between F8 blocks and cyclohexane (or TSCH). As a result, H16 parts will be considered as mixed with the solvents. The SAXS pattern should accordingly describe the shape of fluorinated objects in the liquid phase. 
Several predefined models have been experimented in order to simulate the form factor of fluorinated objects dispersed in the hydrogenated medium, however the cylindrical form factor model proved to be the most appropriate (Figure SI3). The characteristic dimensions of cylinders, length and radius, have been taken from literature [2]. In the F8H16 case, it should be a $1.16 \mathrm{~nm}$ long cylinder with a $0.29 \mathrm{~nm}$ radius (see Figure 3c). A satisfying simulation of the form factor of such a cylinder is compared to experimental data in Figure 3a. Figure $3 \mathrm{~d}$ compares the experimental SAXS data of a 20wt. \% mixture of F8H16 in TSCH at two temperatures with simulations involving fluorinated object of different shapes depicted in Figure $3 \mathrm{c}$. The fitting parameters are detailed in Table 1.

In $\mathrm{CH}$, our calculations clearly show that F8H16 molecules are under monomer form. However, calculations for the F8H16/TSCH mixture suggest the formation of larger objects. Using only small cylinders to simulate our experimental data was not possible. Another contribution, described here as some hexamer species, had to be added to the total contribution. These bigger objects can also be simulated by cylinders, as represented in Figure $3 \mathrm{c}$. Then, as temperature decreases, the monomer contribution is progressively reduced as the hexamer one is enhanced. Table 1 reports the data used in the calculations for F8H16.

\begin{tabular}{|c|c|c|c|c|c|c|c|}
\hline & & $\mathbf{T}\left({ }^{\circ} \mathbf{C}\right)$ & Objects & $N\left(* 10^{-24}\right)$ & $\mathbf{R}(\mathbf{n m})$ & L (nm) & $\Delta \rho\left(\mathrm{cm}^{-2}\right)$ \\
\hline \multirow{4}{*}{$\underset{\mathscr{D}}{\mathbb{D}}$} & \multirow{4}{*}{$\begin{array}{c}\mathrm{F} 8 \mathrm{H} 16,20 \% \\
\text { mass fraction in } \\
\text { TSCH } \\
\left(\mathrm{N}_{\mathrm{th}}=177 * 10^{-24}\right)\end{array}$} & \multirow{2}{*}{$50^{\circ} \mathrm{C}$} & A & 140 & 0,3 & 1,2 & \multirow{4}{*}{$7,34 \mathrm{E}+10$} \\
\hline & & & B & 5 & 0,5 & 2,4 & \\
\hline & & \multirow{2}{*}{$28^{\circ} \mathrm{C}$} & A & 30 & 0,3 & 1,2 & \\
\hline & & & $\mathrm{B}$ & 12 & 0,5 & 2,4 & \\
\hline $\boldsymbol{U}$ & $\begin{array}{c}\mathrm{F} 8 \mathrm{H} 16,20 \% \\
\text { mass fraction in } \\
\mathrm{CH} \\
\left(\mathrm{N}_{\mathrm{th}}=158 * 10^{-24}\right)\end{array}$ & $20^{\circ} \mathrm{C}$ & A & 110 & 0.29 & 1.16 & $8,06 \mathrm{E}+10$ \\
\hline
\end{tabular}

Table 1: Simulation parameters used to fit experimental SAXS curves involving F8H16. $N$ and $\mathbf{N}_{\text {th }}$ respectively stand for the fitted and theoretical object number according to equation (1), $R$ for the radius, $L$ for the length and $\Delta \rho$ for the scattering length density.

It is worthy to note that $\mathrm{N}_{\text {th }}$ values, i.e. the theoretical objects numbers, have been calculated from the initial mass of SFA introduced in the samples. A difference between $\mathrm{N}_{\text {th }}$ and $\mathrm{N}$ used in the calculations exists, however, they are still in the same order of magnitude. Despite these small differences, this aggregation model gives a fairly good picture of F8H16 behavior in TSCH: when temperature decreases, F8H16 molecules tend to aggregate in order to form larger objects and minimize their surface contact with the medium. This phenomenon has not been observed in $\mathrm{CH}$, showing once again the lower affinity of $\mathrm{H} 16$ branches with TSCH than with $\mathrm{CH}$.

\section{$\underline{\text { 3/ Aggregation of other SFA in cyclic solvents }}$}

In order to evaluate the effect of the length of the hydrogenated part on the SFA aggregation behavior, we compared F8H16 with a SFA possessing a shorter hydrogenated part: F8H10. Figure SI4a shows that the shortening of the hydrogenated part of the SFA results in a 
decrease of the gel-liquid transition temperature in the pure compound and in TSCH/F8H10 mixtures at any concentration. Same effect is observed in CH/F8H10 mixtures, but the phase diagram is not presented as the transition temperature was out of reach (below $-5^{\circ} \mathrm{C}$ ) at low SFA concentration for our SAXS apparatus.

Figure SI5 shows the SAXS profiles of mixtures of F8H10 in $\mathrm{CH}$ (Figure SI5a) or TSCH (Figure SI5b) at different concentrations and temperatures. It appears that structure factors emerge as soon as the concentration of F8H10 reaches $40 \mathrm{wt}$. \%. Consequently, we focused on the simulation of the experimental data with form factors only on the basis of experimental data obtained on 20 wt.\% of F8H10 in $\mathrm{CH}$ (Figure 4a), and 7 and 20 wt.\% of F8H10 in TSCH (Figure 4b) .

In $\mathrm{CH}$, as shown on Figure 4a and Table 2, the SAXS patterns tend to follow the same trends as previously observed for $\mathrm{F} 8 \mathrm{H} 16$ in $\mathrm{TSCH}$ : at high temperature, $\mathrm{F} 8 \mathrm{H} 10$ molecules are dispersed in the liquid under monomer form. When temperature decreases from $25^{\circ} \mathrm{C}$ down to $-4^{\circ} \mathrm{C}, \mathrm{F} 8 \mathrm{H} 10$ tends to aggregate under trimer form, while the monomer number in solution decreases from $190 \times 10^{-24}$ to $150 \times 10^{-24}$.

In $\mathrm{TSCH}$ however (Figure 4b), the F8H10 under monomer form only exists for low concentration of SFA, i.e. 7 wt.\% in the example. For higher concentrations, the form factor is different. The affinity between the $\mathrm{H} 10$ tails and $\mathrm{TSCH}$ is lower than in $\mathrm{CH}$, promoting the aggregation of F8H10 into larger objects. In order to simulate these objects, several geometrical models have been applied. Finally, flat cylinders, with diameters larger than their length, have been found to give the best agreement with experimental data. Their morphology is depicted in Figure 4c. This reasonable assessment is inspired by some previous works on SFA aggregation $[6,7,14,24]$, describing the formation of gels as the combination of flat objects so as to build the solid skeleton of the structure.
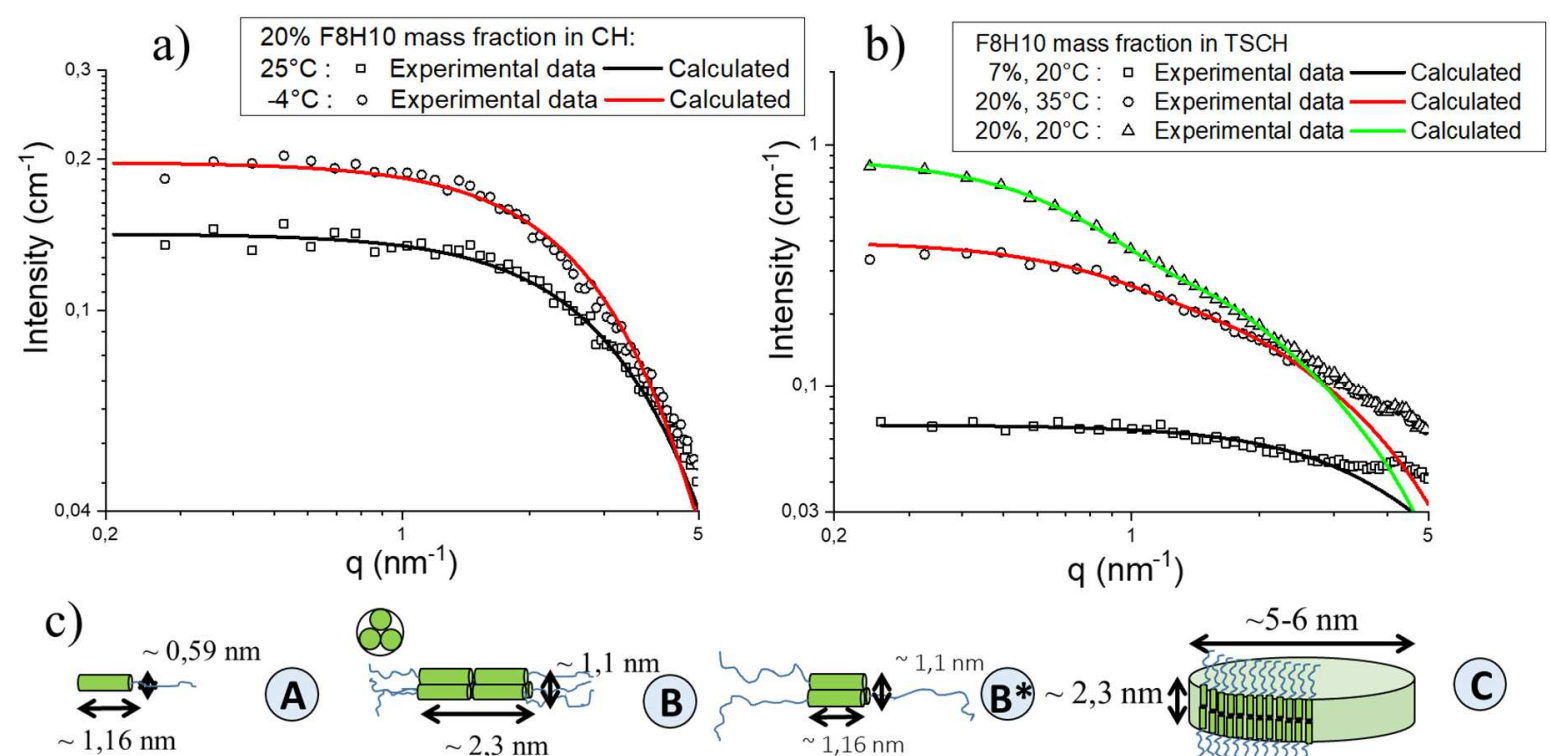

(A)
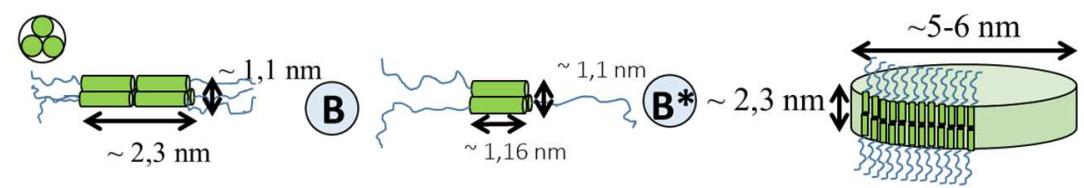

(c)

Figure 4: Experimental and simulated SAXS profiles of mixtures of F8H10 in CH (a) and TSCH (b). Schemes of simulated objects are represented in (c). 


\begin{tabular}{|c|c|c|c|c|c|c|c|}
\hline & & $\mathbf{T}\left({ }^{\circ} \mathbf{C}\right)$ & Objects & $N\left(* 10^{-24}\right)$ & $\mathbf{R}(\mathbf{n m})$ & $\mathbf{L}(\mathbf{n m})$ & $\Delta \rho\left(\mathrm{cm}^{-2}\right)$ \\
\hline \multirow{7}{*}{$\underset{\mathscr{D}}{\tilde{D}}$} & $\begin{array}{c}\mathrm{F} 8 \mathrm{H} 107 \% 20^{\circ} \mathrm{C} \\
(\mathrm{Nth}=77)\end{array}$ & $20^{\circ} \mathrm{C}$ & A & 93 & 0,3 & 1,2 & $7,32 \mathrm{E}+10$ \\
\hline & \multirow{3}{*}{$\begin{array}{c}\mathrm{F} 8 \mathrm{H} 1020 \% 20^{\circ} \mathrm{C} \\
(\mathrm{Nth}=210)\end{array}$} & \multirow{3}{*}{$20^{\circ} \mathrm{C}$} & $\mathrm{A}$ & 60 & 0,3 & 1,2 & \multirow{6}{*}{$7,35 \mathrm{E}+10$} \\
\hline & & & B & 13 & 0,53 & 2,3 & \\
\hline & & & $\mathrm{C}$ & 0,02 & 3,15 & 2,3 & \\
\hline & \multirow{3}{*}{$\begin{array}{c}\mathrm{F} 8 \mathrm{H} 1020 \% 35^{\circ} \mathrm{C} \\
(\mathrm{Nth}=210)\end{array}$} & \multirow{3}{*}{$35^{\circ} \mathrm{C}$} & A & 145 & 0,3 & 1,2 & \\
\hline & & & B & 7,5 & 0,53 & 2,3 & \\
\hline & & & $\mathrm{C}$ & 0,011 & 2,6 & 2,3 & \\
\hline \multirow{3}{*}{$\bar{U}$} & \multirow{3}{*}{$\begin{array}{c}\text { F8H10 20\% } \\
(\mathrm{Nth}=180)\end{array}$} & \multirow{2}{*}{$-4^{\circ} \mathrm{C}$} & A & 150 & 0.3 & 1.2 & \multirow{3}{*}{$8,06 \mathrm{E}+10$} \\
\hline & & & $\mathrm{B}^{*}$ & 10 & 0.55 & 1.2 & \\
\hline & & $25^{\circ} \mathrm{C}$ & $\mathrm{A}$ & 190 & 0.3 & 1.2 & \\
\hline
\end{tabular}

Table 2: Simulation parameters used to fit experimental SAXS curves involving F8H10. $N$ and $\mathbf{N}_{\text {th }}$ respectively stand for the fitted and theoretical object number according to equation (1), $R$ for the radius, $L$ for the length and $\Delta \rho$ for the scattering length density.

The good agreement between experimental and simulated data tend to prove the validity of this model, enlightening the formation of larger flat objects made of 150 F8H10 SFA in $\mathrm{TSCH}$. If the existence of such large aggregates was suspected in these systems, we give a first estimation of their actual size and abundance in the present work. It can be noticed that the proportion of these objects is very small ( 1 per 10000 objects) in such dilute conditions, yet their large volume and discotic shape make their form factor visible by SAXS.

Results on the aggregation of F6Hm will only be presented for SFA/TSCH mixtures, as structures factors were systematically observed in the SAXS patterns of $\mathrm{CH}$ mixtures. The phase diagram drawn in Figure SI4b shows that the decrease of the fluorinated part, even when the hydrogenated part is increased (e.g. comparing F8H10 and F6H14), results in the lowering of the gel transition temperature. This indicates that the length of the fluorinated part of SFA has a stronger impact on the SFA gelation in TSCH with regards to the length of the hydrogenated part. F6H10 binary phase diagram is not represented as its transition temperature was out of reach, except as pure compound, where its first Bragg peak appears at $-3^{\circ} \mathrm{C}$.

In the liquid state, $\mathrm{F} 6 \mathrm{H} 14$, exists under monomeric form at high enough temperatures $\left(>30^{\circ} \mathrm{C}\right)$ in TSCH (see Figure SI6a and Table SI2). Then, as temperature decreases and at low concentration (20 wt\%), F6H14 tends to aggregate under trimer form. At higher concentration (40 wt \%), trimeric objects are formed even at the highest temperature investigated. At lower temperatures, a third kind of aggregates needs to be included in the calculations. A good agreement between experimental and calculated curves has been obtained with some hexamer-type aggregates. However, in this case, the simulations have been applied to a larger concentration range, leading to the consideration of a small structure factor in the calculations to refine results of the $\mathrm{F} 6 \mathrm{H} 1440 \mathrm{wt} \%$ at $40^{\circ} \mathrm{C}$. The corresponding parameters are given in Table SI2. 
Regarding F6H10 species (Figure SI6b and Table SI2), it can be noted that they tend to form larger objects than F6H14 in TSCH, just as we observed comparing F8H16 and F8H10. Moreover, the same kind of large and flats objects made of 150 SFA needs to be used to fit the experimental SAXS data.

At high temperature and low concentration, our results show that SFA can exist under monomer form in the cyclic solvents we tested. Then, as both of these parameters evolve, SAXS data monitor an aggregation phenomenon. Trimer or hexamer cylindrical aggregates have often be used to describe the systems in our calculations. However, the monomer form remains the main species in solution in all studied cases. Investigated FnH10 also show a tendency to form bigger flats objects than F8H16. It has also been shown that the silicon atoms on TSCH rings decrease their affinity with SFA, as the formed objects tend to be larger than in $\mathrm{CH}$ at same temperature and concentration. Another clue to this assumption is the gel formation temperature that is always higher in $\mathrm{TSCH}$ than in $\mathrm{CH}$, illustrating the restrained solubility of SFA in TSCH.

The compared effects of the structure of the SFA molecules on their tendency to form aggregates in cyclic organic solvents has been summarized in Figure 5. For the sake of clarity, only $20 \mathrm{wt} \%$ mixtures were considered. TSCH has been chosen as the solvent because its gelation temperature is reachable for any SFA on the available temperature range. However, some of the results involving TSCH most probably apply also to $\mathrm{CH}$.

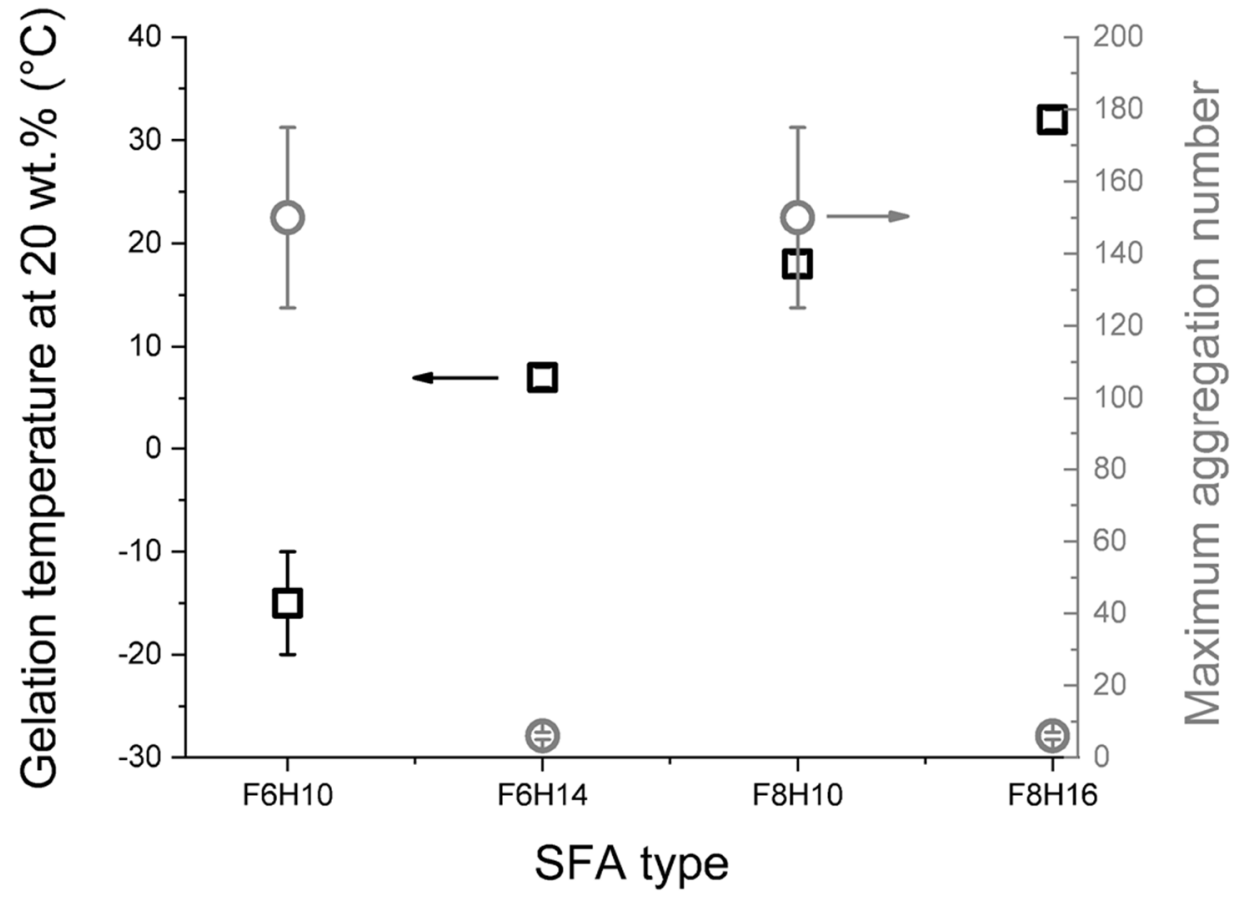

Figure 5: Gelation temperature of 20 wt.\% mixtures (black squares, left axis) and maximum aggregation number in the liquid phase (grey circles, right axis) of F6H10, F6H14, F8H10, and F8H16. 
In Figure 5, SFA are positioned according to the following rule: smaller F6 chains are placed at the left, from the shortest to the longest hydrogenated parts, and the longer F8 chains appear on the right, also from the shortest to the longest hydrogenated chains. It is then obvious that the gelation temperature is mainly guided by the length of the fluorinated part of the SFA, in good agreement with previous results [2,28]. To a lesser extent, the length of the hydrogenated chain also plays a role in the gelation temperature: longer hydrogenated parts result in higher gelation temperature. This can be related to the restrained solubility of large chains in the cyclic solvents. Note that the ratio between the fluorinated part and the hydrogenated part does not appear to be a relevant parameter.

The gelation temperature should however be considered apart from the tendency to form aggregates in the liquid phase. The maximum aggregation numbers observed in the liquid phase has been plotted in Figure 5 (right scale), and it does not follow the same trend than the gelation temperature. For a given length of fluorinated part, shortest hydrogenated chains seem to favor the formation of large aggregates of 150 SFA without immediate transformation of the liquid mixture into gel. The tendency to form large aggregates is therefore not mainly directed by the fluorinated part of SFA, but rather to the length of the hydrogenated chains. This interesting property might originate from the increased mobility of the SFA possessing shorter hydrogenated chains, whose fluorinated parts can stack and form large aggregates without leading the medium to jam.

\section{Conclusion}

From the results we presented on the structure of different SFA considered as pure compounds or mixed with $\mathrm{CH}$ and $\mathrm{TSCH}$, some general tendencies can be drawn concerning the aggregation mechanism of these molecules. SFA aggregation is led by fluorinated parts, while the hydrogenated ones tend to restrict it. The objects formed by the aggregation process appear to be rather small according to our calculations, the majority staying under monomeric form, and a minority aggregating under trimer or hexamer cylindrical forms. Evidences of larger platelet-like objects formation has also been noticed for SFA with short hydrogenated parts (i.e. FnH10). Finally, in all cases, the most important parameters favoring SFA aggregation are temperature and concentration. SFA aggregates will only form in a determined temperature range: under this range, SFA will form gels, while above this range, only monomeric SFA species will be found. The concentration impact is rather simple: the more SFA in solution, the easier the aggregation.

The temperature of gel formation in the different solvents is directly linked to the fluorinated part length, as F8Hm melts at higher temperature than $\mathrm{F} 6 \mathrm{Hm}$. This is in line with the lower solubility of larger fluorinated molecules in a hydrogenated oil. SFA hydrogenated parts seem to have a secondary impact on the mixture melting point: longer $\mathrm{H}$ chains implying higher melting points for a given fluorinated part length. For linear n-alkanes, the evolution of the melting temperature is monotonic [29]. As there is no major conformational effect observed with these SFA molecules, it is not expected that a non-monotonic evolution of melting 
temperatures would occur. Parameters guiding the gel formation also include solvent nature, $\mathrm{CH}$ having more affinity with SFA than TSCH, as it could be expected.

Thanks to these quantitative determination of the size and number of aggregates in mixtures containing different SFA and cyclic solvents, we have been able to discriminate the influence of the fluorinated and hydrogenated part of SFA on their aggregation behavior. These insights in the aggregation and gelation mechanisms of SFA in cyclic organic solvents open interesting new perspectives in the view of designing templated ceramic materials from such mixtures. Whereas macroporous $\mathrm{SiC}$ has been obtained starting from the gel phase of SFA/TSCH mixtures, the control of the nanometric size of SFA aggregated in the liquid phase is likely to result in the achievement of mesoporous $\mathrm{SiC}$.

\section{Acknowledgments}

The authors acknowledge the French National Agency for Research (ANR) for the funding of the FANTA-SiC project (ANR-JS08-0010). Dr D. Meyer, Dr P. Bauduin, and Dr M. Wong Chi Man are thanked for scientific advice.

\section{$\underline{\text { References }}$}

[1] R.J. Twieg, T.P. Russell, R. Siemens, J.F. Rabolt, Observations of a gel phase in binary mixtures of semifluorinated n-alkanes with hydrocarbon liquids, Macromolecules. 18 (1985) 1361-1362. doi:10.1021/ma00148a060.

[2] M.P. Krafft, J.G. Riess, Chemistry, Physical Chemistry, and Uses of Molecular Fluorocarbon-Hydrocarbon Diblocks, Triblocks, and Related Compounds-Unique "Apolar" Components for Self-Assembled Colloid and Interface Engineering, Chem. Rev. 109 (2009) 1714-1792. doi:10.1021/cr800260k.

[3] B.P. Binks, P.D.I. Fletcher, S.N. Kotsev, R.L. Thompson, Adsorption and Aggregation of Semifluorinated Alkanes in Binary and Ternary Mixtures with Hydrocarbon and Fluorocarbon Solvents, Langmuir. 13 (1997) 6669-6682. doi:10.1021/la970408i.

[4] B.P. Binks, P.D.I. Fletcher, W.F.C. Sager, R.L. Thompson, Semifluorinated alkanes as primitive surfactants in apolar hydrocarbon and fluorocarbon solvents, J. Mol. Liq. 72 (1997) 177-190. doi:10.1016/S0167-7322(97)00038-X.

[5] P. Lo Nostro, Phase separation properties of fluorocarbons, hydrocarbons and their copolymers, Adv. Colloid Interface Sci. 56 (1995) 245-287. doi:10.1016/00018686(95)00234-H.

[6] P.L. Nostro, C.Y. Ku, S.-H. Chen, J.-S. Lin, Effect of a Semifluorinated Copolymer on the Phase Separation of a Fluorocarbon/ Hydrocarbon Mixture, J. Phys. Chem. 99 (1995) 10858-10864. doi:10.1021/j100027a029.

[7] C.-Y. Ku, P. Lo Nostro, S.-H. Chen, Structural Study of the Gel Phase of a Semifluorinated Alkane in a Mixed Solvent, J. Phys. Chem. B. 101 (1997) 908-914. doi:10.1021/jp963120v.

[8] A. Runnsjö, V. Kocherbitov, G. Graf, A. Pettigrew, D. Scherer, K. Mortensen, J.

Engblom, Semifluorinated alkanes and alkanes: A phase study of the perfluorohexyloctane - Tetradecane system, J. Chem.Thermodynamics 105 (2017) 352361. doi: 10.1016/j.jct.2016.10.032. 
[9] X. Liu, J.G. Riess, M.P. Krafft, Self-Organization of Semifluorinated Alkanes and Related Compounds at Interfaces: Thin Films, Surface Domains and Two-Dimensional Spherulites, Bull. Chem. Soc. Jpn. 91 (2018) 846-857. doi:10.1246/bcsj.20170431.

[10] B.P. Binks, P.D.I. Fletcher, W.F.C. Sager, R.L. Thompson, Adsorption of Semifluorinated Alkanes at Hydrocarbon-Air Surfaces, Langmuir. 11 (1995) 977-983. doi:10.1021/la00003a047.

[11] M. Broniatowski, P. Dynarowicz-Łątka, Semifluorinated alkanes — Primitive surfactants of fascinating properties, Adv. Colloid Interface Sci. 138 (2008) 63-83. doi:10.1016/j.cis.2007.11.002.

[12] P. Marczuk, P. Lang, A Structural X-ray Study on Semifluorinated Alkanes (SFA): SFA Revisited, Macromolecules. 31 (1998) 9013-9018. doi:10.1021/ma981130j.

[13] L. Tattini, P. Lo Nostro, L. Scalise, B.W. Ninham, P. Baglioni, Gels from a semifluorinated n-alkane in fluorinated solvents as a probe for intermolecular interactions, J. Colloid Interface Sci. 339 (2009) 259-265. doi:10.1016/j.jcis.2009.07.037.

[14] T. Nardin, B. Gouze, J. Cambedouzou, P. Bauduin, M.W.C. Man, X. Deschanels, D. Bourgeois, D. Meyer, O. Diat, Elaboration of porous silicon carbide by soft templating molecular precursors with semi-fluorinated alkanes, J. Mater. Chem. A. 3 (2015) 30823090. doi:10.1039/C4TA05996D.

[15] B. Gouze, Auto organisation de semifluoroalcanes amphiphiles en milieux non-aqueux : vers un carbure de silicium à mésoporosité contrôlée, $\mathrm{PhD}$ manuscript from Ecole nationale supérieure de chimie de Montpellier, 2016. http://www.theses.fr/2016ENCM0002.

[16] N.H. Andersen, C.J. Nielsen, P. Klaeboe, G.A. Guirgis, J.S. Overby, S.M. Askarian, Infrared and Raman spectra, DFT-calculations and spectral assignments of 1,3,5trisilacyclohexane, J. Mol. Struct. $1076 \quad$ (2014) 419-425. doi:10.1016/j.molstruc.2014.07.077.

[17] G. Fritz, E. Matern, The Formation of Carbosilanes, in: Carbosilanes, Springer Berlin Heidelberg, 1986: pp. 5-98. http://link.springer.com/chapter/10.1007/978-3-642-708008_2 (accessed September 1, 2015).

[18] I. Arnason, G.K. Thorarinsson, E. Matern, Conformations of silicon-containing rings: Part 1. A conformational study on 1,3,5-trisilacyclohexane. Comparison of ab initio, semiempirical, and molecular mechanics calculations. Conformational energy surface of 1,3,5-trisilacyclohexane, J. Mol. Struct. THEOCHEM. 454 (1998) 91-102. doi:10.1016/S0166-1280(98)00234-6.

[19] Y. Li, R. Beck, T. Huang, M.C. Choi, M. Divinagracia, Scatterless hybrid metal-singlecrystal slit for small-angle X-ray scattering and high-resolution X-ray diffraction, J. Appl. Crystallogr. 41 (2008) 1134-1139. doi:10.1107/S0021889808031129.

[20] M. Geppi, S. Pizzanelli, C.A. Veracini, C. Cardelli, E. Tombari, P. Lo Nostro, Investigation of the Solid State Behavior of a Semifluorinated n-Alkane by Means of NMR, Calorimetric, and Dielectric Techniques, J. Phys. Chem. B. 106 (2002) 15981605. doi:10.1021/jp0125956.

[21] A. Guinier, G. Fournet, Wiley; New York: 1955, Small Angle Scatt. X-Rays. (n.d.).

[22] J. Kohlbrecher, User guide for the SASfit software package, (2014).

[23] R.F. Fedors, A method for estimating both the solubility parameters and molar volumes of liquids, Polym. Eng. Sci. 14 (1974) 147-154. doi:10.1002/pen.760140211.

[24] P. Lo Nostro, S.H. Chen, Aggregation of a semifluorinated n-alkane in perfluorooctane, J. Phys. Chem. 97 (1993) 6535-6540. doi:10.1021/j100126a033.

[25] F.D. Tsourtou, O. Alexiadis, V.G. Mavrantzas, V. Kolonias, E. Housos, Atomistic Monte Carlo and molecular dynamics simulation of the bulk phase self-assembly of 
semifluorinated alkanes, Chem. Eng. Sci. $121 \quad$ (2015) 32-50. doi:10.1016/j.ces.2014.09.009.

[26] R.-Y. Wang, X.-Y. Liu, J. Narayanan, J.-Y. Xiong, J.-L. Li, Architecture of Fiber Network: From Understanding to Engineering of Molecular Gels, J. Phys. Chem. B. 110 (2006) 25797-25802. doi:10.1021/jp065101j.

[27] P. Marczuk, P. Lang, M. Möller, Bulk structure and surface activity of semifluorinated alkanes, Colloids Surf. Physicochem. Eng. Asp. 163 (2000) 103-113. doi:10.1016/S0927-7757(99)00435-5.

[28] R. Twieg, J.F. Rabolt, An investigation of the raman-active longitudinal acoustical mode (LAM) in semifluorinated n-alkanes, J. Polym. Sci. Polym. Lett. Ed. 21 (1983) 901-906. doi:10.1002/pol.1983.130211107.

[29] J.C.S. Costa, A. Mendes, L.M.N.B.F. Santos, Chain Length Dependence of the Thermodynamic Properties of n-Alkanes and their Monosubstituted Derivatives, J. Chem. Eng. Data 63 (1) (2018), 1-20. doi: 10.1021/acs.jced.7b00837 


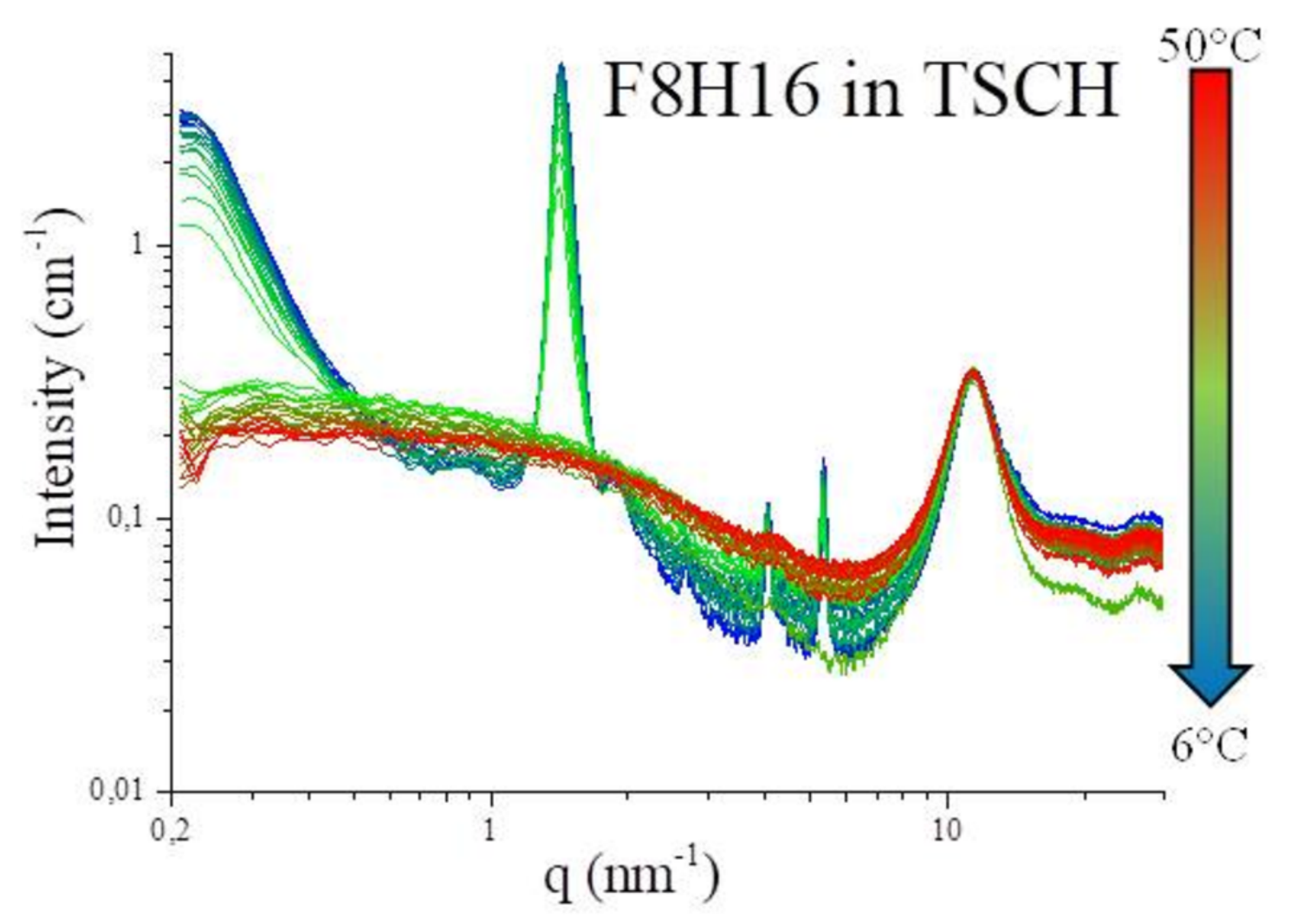

